\title{
Vitiligo as a Köebner phenomenon after oncoplastic breast surgery
}

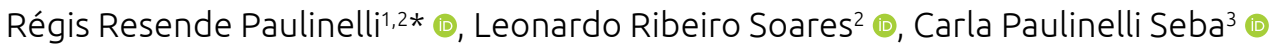

\section{ABSTRACT}

The Köebner phenomenon is characterized by the appearance of several types of dermatological lesions after traumatic stimulation. The triggering of this phenomenon after breast surgery is uncommon and usually associated with psoriatic lesions. The aim of this study was to describe two cases of vitiligo as the initial manifestation of Köebner phenomenon after breast oncoplastic surgery. Case 1: female, 41 years old, no history of dermatological pathologies, presenting with tubular carcinoma in the right breast. Quadrantectomy and sentinel lymph node biopsy were performed, followed by reconstruction with mammoplasty. Later, the patient started on tamoxifen and underwent radiotherapy, without complications. Thirty days after treatment, the patient presented progressive depigmentation of the areola-papillary complex. Topical treatment was started with dermatological ointment tacrolimus monohydrate and, after one year, the condition was completely resolved. Case 2: 52-yearold woman with previous history of vitiligo on the face, with complete clinical response after dermatological treatment. She was diagnosed with ductal carcinoma in situ on the left breast and underwent quadrantectomy, by means of mammoplasty using the round block technique. Afterwards, she underwent radiotherapy and started tamoxifen. Four years after the surgery, she developed dyschromia in the ipsilateral periareolar region and was diagnosed with vitiligo. Local dermopigmentation was offered, but the patient opted for an expectant conduct and clinical follow-up. To our knowledge, this is the first description of Köebner phenomenon after breast oncoplastic surgery. In these cases, the therapeutic approach must be multidisciplinary and count on the assessment of multiple clinical and individual parameters.

KEYWORDS: breast neoplasms; vitiligo; conservative treatment; breast cancer; oncoplasty.

\section{INTRODUCTION}

The first description of the Köebner phenomenon, in 1877, involved psoriatic lesions secondary to trauma in non-affected skin portions of patients with psoriasis.1. The concept of the Köebner phenomenon has been expanded to currently encompass the appearance of several types of skin lesions after local traumatic stimulus, even in individuals with no previously diagnosed dermatological diseases ${ }^{2}$. Although it can affect up to $25 \%$ of psoriasis patients submitted to skin traumatic stimulation, the etiology and pathological mechanisms underlying the phenomenon have not been completely clarified ${ }^{2}$.

In the framework of dermatological lesions that can be triggered by this phenomenon, vitiligo lesions also stand out. Vitiligo is characterized as an acquired disorder that progresses with chronic changes in the pigmentation of the skin and fanera, due to the functional loss of melanocytes ${ }^{3}$. The etiology of vitiligo is still not completely elucidated, although there are autoimmune and genetic components capable of activating the disease, as well as epigenetic features capable of triggering the disease by means of environmental factors. ${ }^{4}$.

Surgical trauma is an environmental factor that can compete with an area of depigmentation in a region of previously normal skin $^{5}$. The development of vitiligo after abrasions, incisions or surgical wounds is known as an isomorphic phenomenon and can happen in patients with a previous diagnosis of the disease. It can, however, also affect patients not diagnosed with vitiligo, at a lower incidence ${ }^{6}$. 
Although the Köebner phenomenon is relatively common in the surgical field, reports of its occurrence after breast surgery are scarce in the literature. In addition, it is usually associated with the occurrence of psoriatic lesions, which makes its presentation in the form of vitiligo even more unusual ${ }^{4,7}$. Thus, the objective of this study was to describe two cases of vitiligo as an initial manifestation of the Köebner phenomenon after breast oncoplastic surgery.

\section{CASE REPORTS}

\section{Case 1}

A 52-year-old female, who had been using hormone therapy for three years, was admitted to the service due to altered exams. History of vitiligo on the face, with complete clinical response after dermatological treatment. Upon physical examination, no palpable change was felt in the breasts and armpits. Mammography showed amorphous microcalcifications grouped in the upper lateral quadrant of the left breast. left breast mammotomy was performed and the anatomopathological examination showed two foci of ductal carcinoma in situ, measuring 0.3 and $0.4 \mathrm{~cm}$, respectively.

Immunohistochemistry of the lesion revealed expression of estrogen (2+/4+) and progesterone (1+/4+), Ki67 receptors in 5\% of neoplastic cells and absence of HER2 oncoprotein. Left quadrantectomy was performed by means of mammoplasty using the round block technique and, following the location of the metal clip inserted during the mamotomy, no residual neoplasia was found (pTis cNO M0, Ec 0). The patient had good postoperative recovery and satisfactory breast symmetry. Then, she underwent adjuvant radiotherapy on the left breast and started using Tamoxifen, not showing any serious adverse events. Four years after surgery, she developed dyschromia in the left breast's periareolar region, which was diagnosed as vitiligo in a dermatological consultation. The patient was offered the possibility of local dermopigmentation, but opted for an expectant conduct and clinical follow-up (Figure 1).

\section{Case 2}

Female 41-year-old patient with no history of breast surgery or previous dermatological diseases, reported having a nodule in her right breast for two years in progressive growth. Upon physical examination, no palpable change was felt in the breasts and armpits. Breast ultrasound showed simple bilateral cysts and a hypoechoic, lobulated nodule measuring $0.7 \mathrm{~cm}$ in the lower medial quadrant of the right breast. Mammography showed punctiform microcalcifications grouped in the same topography of the right breast, which seemed stable in relation to previous mammographic exams. The lesion was removed and identified as tubular carcinoma grade I, measuring $1.1 \mathrm{~cm}$ and touching the surgical margins. The patient underwent quadrantectomy and sentinel lymph node biopsy on the right breast, with immediate reconstruction, using J mammoplasty. The anatomopathological study showed absence of residual neoplasia and free axillary lymph nodes (pTlc pN0sn M0, Ec Ia). Immunohistochemistry of the lesion revealed expression of estrogen $(3+/ 4+)$ and progesterone $(1+/ 4+)$, negative HER 2 and Ki67 receptors in $5 \%$ of neoplastic cells. The patient had a good postoperative recovery and satisfactory breast symmetry. Afterwards, she started adjuvant endocrine therapy with Tamoxifen and adjuvant radiotherapy, which was uneventful. Thirty days after radiotherapy, the patient presented with progressive depigmentation of the areola-papillary complex on the right (Figure 2). The patient was offered the possibility of local dermopigmentation, but opted for topical treatment with tacrolimus monohydrate dermatological ointment $0.1 \%$ twice a day. After six months of treatment, she had a partial improvement of hypochromia in the right breast (Figure 3).

\section{DISCUSSION}

The Köebner phenomenon after breast surgery is uncommon and generally associated with the occurrence of psoriatic lesions ${ }^{2,7}$; however, there are descriptions of the phenomenon after radical mastectomy ${ }^{8}$, bilateral prophylactic
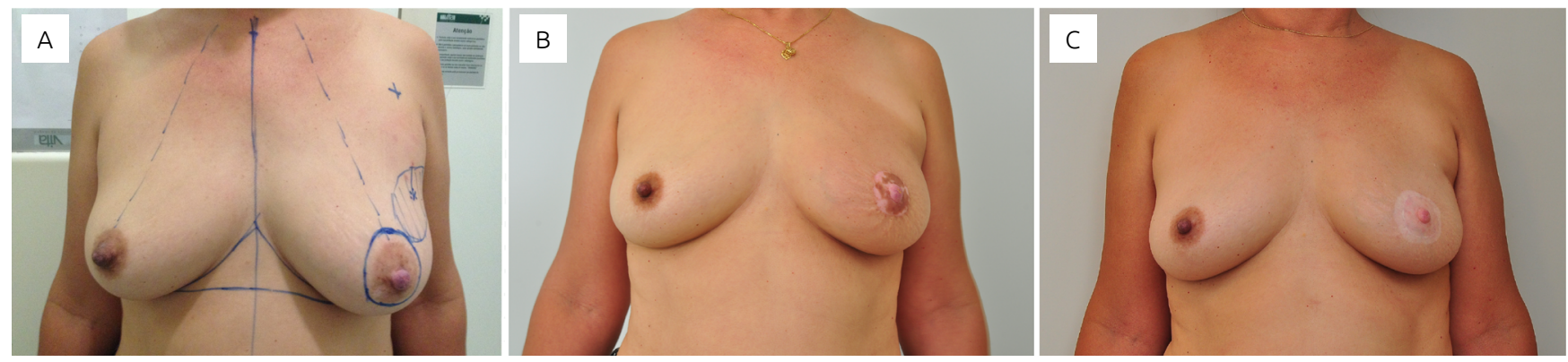

Figure 1. Case 1: (A) Preoperative marking. (B) Köebner phenomenon in the postoperative period of oncoplastic surgery, six months after radiotherapy. (C) Late residual appearance two years after surgery. 
mastectomy and reconstruction with prostheses ${ }^{7}$, and after skin-sparing mastectomy with immediate reconstruction, using prosthesis and latissimus dorsi muscle flap 9 . To our knowledge, the cases reported in the current study are the first descriptions of this phenomenon after breast oncoplastic surgery. In this context, the early recognition of the condition by the professional surgeon can lead to the adequate therapeutic management and, possibly, to more satisfactory clinical results.

The pathophysiology underlying the Köebner phenomenon remains inconclusive, despite the frequent observation of epidermal cell damage associated with the inflammatory dermal reaction ${ }^{2,7}$, but experimental studies involving its induction have shown divergent results when it comes to the clinical manifestations of the lesions ${ }^{2}$. Thus, physical, biochemical, and immunological factors can also be associated with the occurrence of the Köebner phenomenon and contribute to the diversity of clinical presentations seen in the literature ${ }^{2,4,10}$.

Radiotherapy is also associated with several clinical manifestations, as well as early and late skin toxicity ${ }^{11,12}$, including the occurrence of the phenomenon in the absence of previous surgical procedures ${ }^{13}$. However, the occurrence of vitiligo after radiotherapy is uncommon and, to our knowledge, there are less than 20 cases reported worldwide ${ }^{12,14}$. The pathophysiology would probably involve the susceptibility of certain melanocytes to apoptosis mediated by oxidative stress, and to free radicals generated by irradiation ${ }^{11-14}$, although most cases report lesions in the entire portion affected by radiotherapy $y^{11,14}$, and not only in scar topography. In addition, the patients described in this series had good tolerance to radiotherapy and minimal inflammatory effect on the breasts, which reduced the possibility of skin lesions secondary to radiotherapy.

As for skin treatment, the severity, topography and clinical presentation of the lesions must be considered. When lesions present in the form of vitiligo, topical treatment with corticosteroids or biological therapies, treatments involving some types of light (for example, narrowband UV-B) and systemic medications, along with various skin pigmentation techniques, can be performed ${ }^{15}$. However, in selected cases, expectant conduct ${ }^{16}$ or the combination of two or more therapies can be adopted ${ }^{17}$. In one of the cases described, clinical response with tacrolimus monohydrate dermatological ointment was satisfactory.

\section{CONCLUSION}

To our knowledge, this is the first description of Köebner phenomenon after breast oncoplastic surgery. In these cases, the therapeutic approach must be multidisciplinary and in accordance with the evaluation of multiple clinical and individual parameters.

\section{AUTHORS' CONTRIBUTION}

R. P.: Conceptualization, funding, research, methodology, management, supervision, validation, visualization, writing of the project - review and editing.

L. R.: Conceptualization, funding acquisition, research, methodology, project management, validation, visualization, writing - review and editing.

C. S.: Conceptualization, funding, research, methodology, management, validation, visualization, writing - project review and editing.
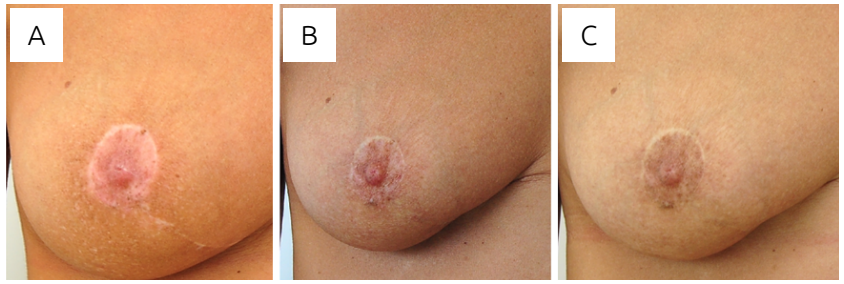

Figure 3. Right breast (A) before and (B) after topical treatment with tracolimus monohydrate dermatological ointment $0.1 \%$, twice a day. Partial improvement in hypochromia after six months of treatment. (C) There was complete improvement after one year of treatment.
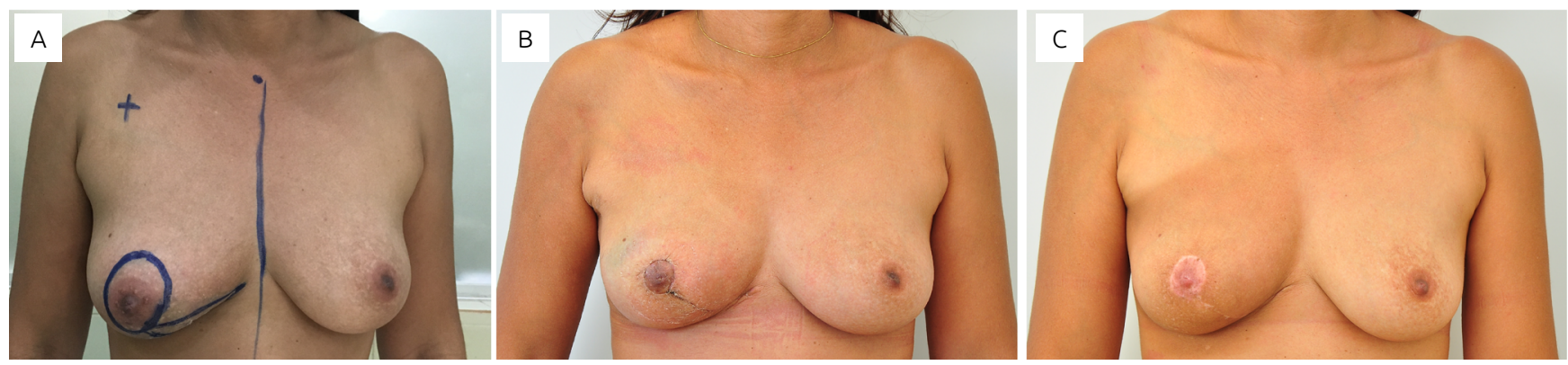

Figure 2. Case 2: (A) Preoperative marking. (B) Immediate postoperative period without dermatological changes two months later. (C) Köebner phenomenon in the late postoperative period of oncoplastic surgery, six months after radiotherapy. 


\section{REFERENCES}

1. Köbner H. Zur Aetiologie Psoriasis. Vjschr Dermatol. 1876;3:559.

2. Weiss G, Shemer A, Trau H. The Koebner phenomenon: review of the literature. J Eur Acad Dermatol Venereol. 2002;16(3):2418. https://doi.org/10.1046/j.1473-2165.2002.00406.x

3. Ghafourian E, Ghafourian S, Sadeghifard N, Mohebi R, Shokoohini Y, Nezamoleslami S, et al. Vitiligo: Symptoms, Pathogenesis and Tratment. Int J Immunopathol Pharmacol. 2014;27(4):485-9. https://doi.org/10.1177/039463201402700403

4. Ji YZ, Liu SR. Koebner phenomenon leading to the formation of new psoriatic lesions: evidences and mechanisms. Biosci Rep. 2019;39(12):BSR20193266. https://doi.org/10.1042/BSR20193266

5. Ganguly AK, Laghimsetty S, Bhagyalakshmi N. Koebner Phenomenon Triggered by External Dacryocystorhinostomy Scar in a Patient With Psoriasis: A Case Report and Literature Review. Ophthalmic Plast Reconstr Surg. 2018;34(2):e52-e53. https://doi.org/10.1097/IOP.0000000000001016

6. Mulekar SV, Asaad M, Ghwish B, Al Issa A, Al Eisa A. Koebner Phenomenon in Vitiligo: Not Always an Indication of Surgical Failure. Arch Dermatol. 2007;143(6):799-816. https://doi. org/10.1001/archderm.143.6.801

7. Alolabi N, White CP, Cin AD. The Koebner phenomenon and breast reconstruction: Psoriasis eruption along the surgical incision. Can J Plast Surg. 2011;19(4):143-4. https://doi. org/10.1177/229255031101900411

8. Bernstein EF, Kantor GR. Treatment-resistant psoriasis due to a mastectomy sleeve: an extensive Koebner response. Cutis. 1992;50(1):65-7.

9. Behranwala KA, Gui GPH. The Koebner phenomenon in a myocutaneous flap following immediate breast reconstruction. Br J Plast Surg. 2002;55:267-8. https://doi.org/10.1054/bjps.2002.3807
10. Ji YZ, Liu SR. Koebner phenomenon leading to the formation of new psoriatic lesions: evidences and mechanisms. Biosci Rep. 2019;39(12). https://doi.org/10.1042/BSR20193266

11. Wu CC, Wang S, AnJJ,SmithDR, Chin C,JadejaPH, etal.Koebner phenomenon: Consideration when choosing fractionation for breast irradiation. Adv Radiat Oncol. 2018;3(2):108-110. https:// doi.org/10.1016/j.adro.2017.11.004

12. Dalmasso C, Tournier É, de Lafontan B, Modesto A, Dalenc F, Chantalat É, et al. Uncommon dermatologic disorders triggered by radiation therapy of breast cancer: A case-series. Cancer Radiother. 2017;21(3):216-221. https://doi.org/10.1016/j. canrad.2016.11.004

13. Charalambous H, Bloomfield D. Psoriasis and radiotherapy: exacerbation of psoriasis following radiotherapy for carcinoma of the breast (the Koebner phenomenon). Clin Oncol (R Coll Radiol). 2000;12(3):192-3. https://doi.org/10.1053/ clon.2000.9149

14. Weitzen R1, Pfeffer R, Mandel M. Benign lesions in cancer patients: Case 3. Vitiligo after radiotherapy for breast cancer in a woman with depigmentation disorder. J Clin Oncol. 2005;23(3):644. https://doi.org/10.1200/JCO.2005.03.078

15. Whitton ME, Pinart M, Batchelor J, Leonardi-Bee J, González $\mathrm{U}$, Jiyad Z, et al. Interventions for vitiligo. Cochrane Database Syst Rev. 2015;(2):CD003263. https://doi.org/10.1002/14651858. CD003263.pub5

16. Dowlen H, Owers K. Koebner phenomenon following steroid injection for trigger finger. J Hand Surg. 2011;36(6):517. https:// doi.org/10.1177/1753193411409132

17. Ezzedine K, Whitton M, Pinart M. Interventions for Vitiligo. JAMA. 2016;316(16):1708-9. https://doi.org/10.1001/ jama.2016.12399 